\title{
COMUNICACIÓN NO VERBAL DEL DOCENTE Y MOTIVACIÓN DE LOGRO EN ALUMNOS DE LA INSTITUCIÓN EDUCATIVA FORTUNATO ZORA CARVAJAL, TACNA 2016.
}

\author{
NON VERBAL COMMUNICATION OF THE TEACHER AND MOTIVATION OF \\ ACHIEVEMENT IN STUDENTS OF THE EDUCATIONAL INSTITUTION \\ FORTUNATO ZORA CARVAJAL, TACNA
}

\section{Resumen}

El objetivo de investigación es determinar la correlación entre la comunicación no verbal del docente y la motivación de logro escolar de los alumnos de la I.E. Fortunato Zora Carbajal. Es una investigación básica, con diseño descriptivo correlacional, la muestra está compuesta por 29 docentes y 121 alumnos; se utilizó como instrumentos un inventario de observación de 20 ítems para la motivación de logro y para la comunicación no verbal un cuestionario con 18 reactivos, ambos instrumentos fueron validados y sometidos a pruebas de confiabilidad, obteniendo 0.74 puntos y 0.77 puntos con el método de alfa de cronbach. Los resultados reportan un $38.02 \%$ de docentes con nivel regular de comunicación no verbal, el análisis de dimensiones reporta para el paralenguaje 3.2 puntos, la expresión kinésica 3.11 puntos, la expresión proxémica 2.90 puntos y la expresión cronémica 2.68 puntos. También se encontró que el $45.5 \%$ de los alumnos presentan un nivel regular de motivación de logro, el análisis dimensional: la tarea obtiene 5.86 puntos, esfuerzo 6.55 puntos, capacidad 5,77 puntos y evaluación 5.51 puntos, en razón de la escala. La hipótesis se comprobó con el coeficiente de correlación Pearson, reportando un $r>0.5$ por tanto, se rechaza $\mathrm{Ho}$ y se acepta la $\mathrm{H} 1$; queda comprobado que existe una asociación lineal positiva significativa a un nivel de $r=0.908$. En conclusión, queda comprobado que existe una correlación significativa entre la comunicación no verbal y la motivación de logro.

Palabras claves: Motivación de logro, comunicación no verbal.
JANYS VANESSA LOURDES PORTOCARRERO GARCÍA 1

\section{Abstract}

The research object is to determine the correlation between the nonverbal communication of the teacher and the motivation of school achievement of the students of the I. Fortunato Zora Carbajal. It is a basic research, with correlational descriptive design, the sample is composed of 29 teachers and 121 students; We used as an instrument an inventory of 20 items for motivation of achievement and for nonverbal communication a questionnaire with 18 reagents, both instruments were validated and tested for reliability, obtaining 0.74 points and 0.77 points with the alpha method Of cronbach. The results report a $38.02 \%$ of teachers with regular level of nonverbal communication, dimension analysis reports for paralanguage 3.2 points, kinetic expression 3.11 points, proxemic expression 2.90 points and chronemic expression 2.68 points. It was also found that $45.5 \%$ of the students present a regular level of achievement motivation, dimensional analysis: the task gets 5.86 points, effort 6.55 points, capacity 5.77 points and assessment 5.51 points, due to the scale. The hypothesis was checked with the Pearson correlation coefficient, reporting a $r>0.5$ therefore, Ho is rejected and $\mathrm{H} 1$ is accepted; It is verified that there is a significant positive linear association at a level of $r=0.908$. In conclusion, it is verified that there is a significant correlation between nonverbal communication and motivation of achievement.

Keywords: Achievement motivation, nonverbal communication

1 MAGISTTER EN DOCENCIA UNIVERSITARIA Y GESTIÓN EDUCATIVA 


\section{INTRODUCCIÓN}

Cuando un docente se dirige a los alumnos, acompaña o alterna con lo estrictamente lingüístico de su discurso, apoya o contradice los mensajes transmitidos por sus palabras con la entonación, y utiliza también otros elementos sutilmente estructurados, como la kinésica y el paralenguaje", adicionalmente actúan los signos proxémicos y cronémicos. Por eso para ser un docente eficaz, es fundamental ser un buen comunicador y ello en el doble papel de emisor y receptor de mensajes. Una enseñanza eficaz requiere de una adecuada "competencia comunicativa" en los profesores y persigue, como un objetivo básico, el desarrollo de habilidades comunicativas en los alumnos. Esta competencia estaría asentada en disponer de unos adecuados conocimientos sobre Teoría de la Comunicación y sobre los procesos de interacción didáctica y en la posesión de las actitudes, destrezas y habilidades prácticas necesarias para poder llevar a cabo una comunicación eficaz que tiene que ver con saber manejar adecuadamente la comunicación no verbal.

Tradicionalmente ha existido una separación entre los aspectos cognitivos y los afectivo motivacionales a la hora de estudiar su influencia en el aprendizaje, en la actualidad, existe un creciente interés en estudiar ambos tipos de componentes de forma integrada. "Se puede afirmar que el aprendizaje se caracteriza como un proceso cognitivo y motivacional a la vez" en consecuencia, para aprender es imprescindible "poder" hacerlo, lo cual hace referencia a las capacidades, los conocimientos, las estrategias, y las destrezas necesarias (componentes cognitivos), pero además es necesario "querer" hacerlo, tener la disposición, la intención y la motivación suficientes (componentes motivacionales). Los especialistas más destacados en este tema opinan que, para tener buenos resultados académicos, los alumnos no solo necesitan habilidad, también "voluntad", es decir, control del proceso psicológico que determina la manera de enfrentar y realizar las actividades, tareas educativas. Esto contribuye para que el alumno/a participe en el aprendizaje de una manera más o menos activa, dedique y distribuya su esfuerzo en un período de tiempo, se plantee el logro de un aprendizaje de calidad.

Keil G. (2011) refiere que la existencia de la enseñanza y aprendizaje está ligada a características que fortalecen el vínculo dialogal y pedagógico u otras que la que obstaculizan.
Arcaya H. (2008), afirma que si bien cada sujeto tiene un estilo preferente de aprendizaje, los estilos de enseñanza tienen un impacto en el perfil de los estudiantes, y que en las clases interactivas, los estudiantes mantienen un estilo más colaborativo, participante e independiente que en las clases formales, que promueven estilos competitivos, dependientes y esquivos. Kishimoto B. (2010), refiere que la tecnología y la ciencia desencadenan procesos de transformación global en toda sociedad y está en manos de la pedagogía el acercar estas nuevas herramientas de aprendizaje para construir una cultura que se desenvuelva con comodidad., sin dejar de cultivar los valores humanos. Cerezo M. (2012), establece una relación significativa entre el nivel de capacidad de comunicación corporal del docente y el interés que se despierta en los alumnos por aprender los temas que se desarrollan en clase. Fiske (1982) define a la comunicación como una "interacción social por medio de mensajes". Por su parte, Antonio Pasquali (1978) afirma que "la comunicación aparece en el instante mismo en que la estructura social comienza a configurarse, justo como su esencial ingrediente estructural, y que donde no hay comunicación no puede formarse ninguna estructura social". Quienes se comunican deben tener un grado mínimo de experiencia común y de significados compartidos Hervás (1999). Berlo (1979) afirma que nos comunicamos para influir y para afectar intencionalmente a otras personas. El lenguaje corporal, es un proceso sensorial desde la perspectiva de la percepción y de la emisión. Por ejemplo, la memoria, la concentración, la voluntad, la inteligencia y la creatividad se expresan corporalmente (Günther Rebel, 1995).

La educación supone situaciones comunicativas y fenómenos colectivos en los que la lingüística, la paralingüística y los aspectos kinésicos son importantes para explorar el modo en que se elabora el aprendizaje. El lenguaje y las conductas no verbales entre el maestro y de los alumnos son mediadores para el intercambio de información y para la alimentación de los procesos de conocimiento. En situaciones, en las que los alumnos van a tener que hablar en público, es fundamental que los alumnos hayan trabajado la expresión oral pero igualmente importante es trabajar la entonación, el ritmo, la intensidad y modulación de la voz, etc. para, no sólo comunicar, sino también convencer, impresionar, captar la atención de su público. La 
enseñanza/aprendizaje de los sistemas no verbales es esencial. (Pradelli, 2011, p.103). Gento (1999) sugiere que en la relación entre alumno y profesor, se pueden estudiar diferentes factores de comunicación no verbal. Estos factores se pueden clasificar en dos bloques: elementos humanos y elementos no humanos. Delamont (2011) asegura que el aula con su colocación de mesas, las estructuras temporales, el modo de hablar, las relaciones entre profesores y alumnos, etc., comunica un mensaje antes de que el profesor diga una sola palabra. Según Miller, P.W. (2005): cuando los profesores exhiben mensajes verbales que están en conflicto con los mensajes no-verbales, los alumnos se quedan confusos, y esta confusión muchas veces afecta sus actitudes y su aprendizaje. Los principales elementos de la voz, Pradelli (2011), se observa el timbre, ritmo, entonación, volumen, silencios y pausas

Las habilidades sociales juegan un papel importante en la educación. Es fundamental educar a los niños en un control de los sentimientos ya que esto les ayudará a mejorar las relaciones con el resto de compañeros y con la gente con la que conviva en general. (Ekman, 1969). Respecto a la motivación, González (2003), coincide en decir que ésta es el conjunto de procesos implicados en la activación, dirección y persistencia de la conducta.La motivación ha sido una variable con una gran trascendencia, debido a que cualquier modelo de aprendizaje se centra o conlleva explícita o implícitamente una

\section{OBJETIVOS}

a). Caracterizar la comunicación, el nivel de motivación no verbal de los docentes de la Institución Educativa Fortunato Zora Carvajal de la ciudad de Tacna. b). Relacionar la comunicación no verbal del docente con la motivación de logro que presentan los alumnos.

\section{METODOLOGÍA}

La presente investigación es de tipo básica o pura, de descriptivo - correlacional. El ámbito de la investigación se limitó a los aspectos psicopedagógicos de los alumnos y docentes de educación secundaria de la institución educativa Fortunato Zora Carvajal de la ciudad de Tacna, la misma, que se desarrolló en el año 2016. Se trabajó con 29 docentes y una muestra representativa de 121 alumnos. Se aplicó el inventario de observación, el cual, está compuesto por 20 ítems de evaluación organizados en 4 indicadores (Expresión kinésica, proxémica, paralenguaje y cronémica), con una escala de evaluación de 5 niveles que van desde muy buena hasta muy deficiente comunicación no verbal. Para recoger los datos de la motivación de logro se aplicó como instrumento un cuestionario con alternativas de respuesta mediante el diferencial semántico, el instrumento contiene 18 reactivos que responden a 4 factores de evaluación adaptados de Manassero y Vásquez (1998). La escala de evaluación se mueve en 5 niveles que van desde muy alta motivación de logro hasta muy baja motivación de logro. La confiabilidad del cuestionario de comunicación no verbal fue de 0,740 y la del cuestionario de motivación de logro de 0.775 , según alfa de Cronbach.

\section{RESULTADOS}

En la tabla $N^{\circ} 01$ se presentan los resultados sobre la dimensión del paralenguaje de la comunicación no verbal desde la perspectiva de los alumnos, donde apreciamos : el 0\% muy mala, el $19,01 \%$ mala, el $37.19 \%$ regular, el $36.36 \%$ buena y el $7.44 \%$ muy buena. Respecto a la expresión kinésica de la comunicación no verbal, se observó que el 19,83\% la percibe como mala, el $49.59 \%$ regular, el $24.79 \%$ buena y el $5.79 \%$ muy buena (Tabla 02). Respecto a la dimensión de expresión proxémica de la comunicación no verbal; se observa que el $3.3 \%$ es tiene una percepción muy mala, el 21,5\% mala, el $50.4 \%$ regular, el $24.8 \%$ buena y el $0.0 \%$ muy buena (Tabla 03). Según la dimensión de expresión cronémica de la comunicación no verbal; el 5.8\% la percibe como muy mala, el $33,9 \%$ mala, el $32.2 \%$ regular, el $19 \%$ buena y el $9.1 \%$ muy buena (Tabla 04).Los resultados sobre el nivel de comunicación no verbal, percibida por los alumnos, finalmente es percibida como mala en el $27.27 \%$, el $38.02 \%$ regular, el $34.71 \%$ bueno y el 05 muy bueno (Tabla 05). 
Tabla 1

Dimensión: paralenguaje

\begin{tabular}{ccc} 
& & \\
& Frecuencia & $\%$ \\
\hline Muy mala & 0 & 0.0 \\
\hline Mala & 23 & 19,01 \\
\hline Regular & 45 & 37,19 \\
\hline Bueno & 44 & 36,36 \\
\hline Muy bueno & 9 & 7,44 \\
\hline Total & 121 & 100,0 \\
\hline
\end{tabular}

Tabla 2

Dimensión: expresión kinésica

\begin{tabular}{ccc}
\hline & Frecuencia & $\%$ \\
\hline Mala & 24 & 19,83 \\
\hline Regular & 60 & 49,59 \\
\hline Bueno & 30 & 24,79 \\
\hline Muy bueno & 7 & 5,79 \\
\hline Total & 121 & 100,0 \\
\hline
\end{tabular}

Tabla 3

Dimensión: expresión proxémica

\begin{tabular}{ccc}
\hline & Frecuencia & $\%$ \\
\hline Muy malo & 4 & 3,3 \\
\hline Mala & 26 & 21,5 \\
\hline Regular & 61 & 50,4 \\
\hline Bueno & 30 & 24,8 \\
\hline Muy bueno & 0 & 0.0 \\
\hline Total & 121 & 100,0 \\
\hline
\end{tabular}

Tabla 4

Dimensión: cronémica

\begin{tabular}{ccc}
\hline & Frecuencia & $\%$ \\
\hline Muy malo & 7 & 5,8 \\
\hline Mala & 41 & 33,9 \\
\hline Regular & 39 & 32,2 \\
\hline Bueno & 23 & 19,0 \\
\hline Muy bueno & 11 & 9,1 \\
\hline Total & 121 & 100,0 \\
\hline
\end{tabular}

Tabla 5

Nivel de comunicación no verbal, según la evaluación de los alumnos

\begin{tabular}{ccc}
\hline & Frecuencia & $\%$ \\
\hline Muy mala & 00 & 0.0 \\
\hline Mala & 33 & 27,27 \\
\hline Regular & 46 & 38,02 \\
\hline Bueno & 42 & 34,71 \\
Muy bueno & 00 & 0.0 \\
\hline Total & 121 & 100,0 \\
\hline
\end{tabular}

Respecto a la motivación de logro escolar, relacionada con la dimensión: atribución a la Tarea de la motivación de logro de los alumnos; los resultados indican que el $11.6 \%$ presenta baja motivación, el $65.3 \%$ regular, el $23.1 \%$ alta y el $0 \%$ muy alta (Tabla 06). Según la dimensión: atribución al esfuerzo de la motivación de logro de los alumnos; Los resultados indican el $10.7 \%$ presenta baja motivación, el $37.2 \%$ regular, el $31.4 \%$ alta y el $20.7 \%$ muy alta Tabla 7 ). Respecto la dimensión: atribución a la capacidad de la motivación de logro de los alumnos; el 30.6\% percibe baja motivación, el 33.9\% regular, el $32.2 \%$ alta y el $3.3 \%$ muy alta (Tabla 8 ). Según la dimensión: atribución a la evaluación del docente de la motivación de logro de los alumnos; los resultados indican que el $29.8 \%$ presentan baja motivación, el $43.0 \%$ regular, el $20.6 \%$ alta y el $6.6 \%$ muy alta (Tabla 9). Respecto al nivel de motivación de logro de los alumnos, se aprecia que el $12.4 \%$ manifiesta bajo nivel, el $45.5 \%$ regular, el $41.3 \%$ alto y el $0.8 \%$ muy alto nivel (Tabla 10).
Tabla 6:

Dimensión: atribución a la Tarea

\begin{tabular}{ccc}
\hline & Frecuencia & $\%$ \\
\hline Muy baja & 0 & 0.0 \\
\hline Baja & 14 & 11,6 \\
\hline Regular & 79 & 65,3 \\
\hline Alta & 28 & 23,1 \\
\hline Muy alta & 0 & 0.0 \\
\hline Total & 121 & 100,0 \\
\hline & &
\end{tabular}

Tabla 7:

Dimensión: atribución al esfuerzo

\begin{tabular}{ccc}
\hline & Frecuencia & $\%$ \\
\hline Muy baja & 0 & 0.0 \\
\hline Baja & 13 & 10,7 \\
\hline Regular & 45 & 37,2 \\
\hline Alta & 38 & 31.4 \\
\hline Muy alta & 25 & 20.7 \\
\hline Total & 121 & 100,0 \\
\hline & &
\end{tabular}

Tabla 8:

Dimensión: atribución a la capacidad

\begin{tabular}{ccc}
\hline & Frecuencia & $\%$ \\
\hline Muy baja & 0 & 0,0 \\
\hline Baja & 37 & 30,6 \\
\hline Regular & 41 & 33,9 \\
\hline Alta & 39 & 32.2 \\
\hline Muy alta & 4 & 3,3 \\
\hline Total & 121 & 100,0 \\
\hline
\end{tabular}


Tabla 9:

Dimensión: atribución a la evaluación del docente

\begin{tabular}{ccc}
\hline & Frecuencia & $\%$ \\
\hline Muy baja & 0 & 0,0 \\
\hline Baja & 36 & 29,8 \\
\hline Regular & 52 & 43,0 \\
\hline Alta & 25 & 20.6 \\
\hline Muy alta & 8 & 6,6 \\
\hline Total & 121 & 100,0
\end{tabular}

Tabla 10:

Nivel de motivación de logro

\begin{tabular}{ccc}
\hline & Frecuencia & $\%$ \\
\hline Bajo & 15 & 12,4 \\
\hline Regular & 55 & 45,5 \\
\hline Alto & 50 & 41,3 \\
\hline Muy alto & 1 &, 8 \\
\hline Total & 121 & 100,0 \\
\hline
\end{tabular}

Conforme a los resultados se encontró una asociación lineal positiva significativa entre la motivación de logro y la comunicación no verbal de los docentes de las diferentes áreas curriculares, a un nivel de $r=0.908$ (Fig. 01)

Fig. 1: Nivel de relación motivación/comunicación no verbal

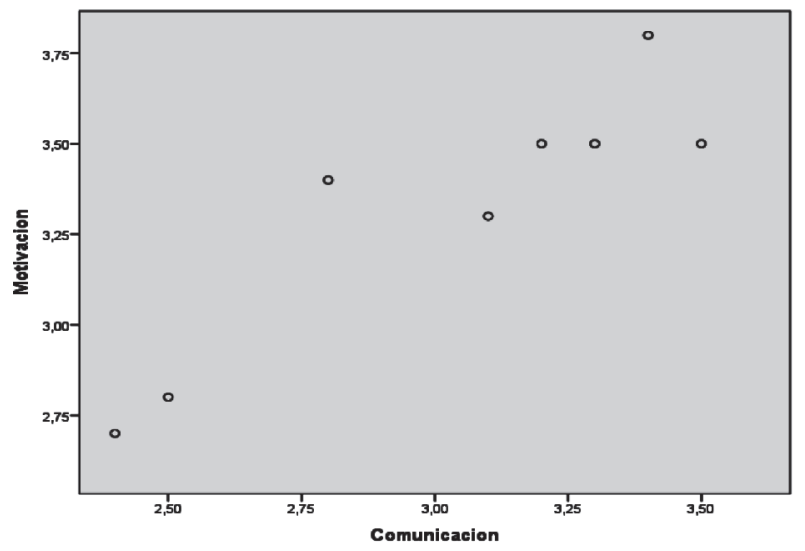

\section{DISCUSIÓN}

Los resultados dan cuenta que la comunicación no verbal de los docentes de las diferentes áreas curriculares no es un factor favorable para motivar a los alumnos con respecto a sus logros escolares, siendo que no logran establecer con éxito una adecuada conexión con los alumnos debido a sus deficiencias de expresión no verbal. Álvarez (2012) como resultado de su investigación concluye que vivimos en una cultura de lo visual. Nuestros alumnos se mueven en un mundo donde las imágenes son más decisivas que los discursos y las palabras. Por ello, los profesores, a través del manejo de la comunicación no verbal, les podemos llegar mejor y de una manera más inmediata, ayudándonos a construir una relación más positiva con ellos y a promover un aprendizaje significativo, que cambie realmente las actitudes y las relaciones. Por su lado Gutiérrez J. (2007) como producto de su investigación afirma que la comunicación no verbal en la interacción alumno-profesor es de gran importancia para comprender y mejorar el proceso de enseñanzaaprendizaje. El/la profesor/a al saber interpretar y manejar las funciones de la comunicación no verbal que cotidianamente las personas emplean, podrá comunicarse con los alumnos de manera más eficaz. García (2014) concluye que la comunicación no verbal posee la capacidad de ir más allá de las palabras y aporta factores que consiguen la empatía necesaria en los procesos de aprendizaje, los mensajes no-verbales que complementan y acompañan al mensaje verbal. Se plantea la necesidad de estudiarlos y analizarlos para que los profesores sean capaces de interpretarlos y ponerlos en práctica con el objetivo de conseguir una comunicación más completa, más directa y más eficaz con los alumnos.

Existe un porcentaje mayoritario de docentes en la Institución Educativa Fortunato Zora Carvajal de la ciudad de Tacna, que presentan una deficiente comunicación no verbal, lo cual se manifiesta en su expresión kinésica, proxémica, cronémica y el paralenguaje. La mayoría de los alumnos muestran bajo nivel de motivación de logro escolar, dado que no muestran mucho interés y no se esfuerzan en la ejecución de las tareas. Se atribuye los resultados al logro de motivación, a que al encontrarse en un entorno que desde pequeños no satisface sus 
necesidades y que no los estimula adecuadamente no se encuentran en las condiciones adecuadas para llevar a cabo las tareas de aprendizaje. Es muy importante el papel que representan los signos no verbales en la adquisición de fluidez comunicativa y todo lo que de ella se deriva, por tanto, es muy importante que los docentes incorporen nuevas habilidades de expresión corporal mediante una capacitación intensiva con el objetivo que los manejos adecuados de estas habilidades logren mejores niveles de motivación en los alumnos. Los directivos de la institución educativa deben promover el feed-back adecuado sobre el estilo de comunicación, tanto en el ámbito verbal como no verbal, mediante distintas vías: grabando nuestra clase y analizándola, abriendo la puerta a la observación de las clases por algunos compañeros, estando atento a las reacciones que muestran los alumnos; de este modo mejorar la expresión kinésica, proxémica, cronémica y el paralenguaje. Hay que concienciar a los educadores de la importancia de la comunicación no verbal en el aula. Muchos educadores tienen una escasa o nula formación en este campo y esto nos debe hacer reflexionar sobre la necesidad prioritaria de realizar cambios en los planes de estudio en la formación del profesorado. Envolver a los estudiantes en una amplia variedad de actividades en donde se fomente la participación, el trabajo cooperativo y se utilice material didáctico diverso y atractivo.

\section{REFERENCIAS BIBLIOGRÁFICAS}

Presentado: Marzo 2017

Aceptado: Junio 2017

Álvarez, Q.(2012). La comunicación no verbal en los procesos de enseñanza-aprendizaje: el papel del profesor. [Tesis de grado]. Universidad de Santiago de Compostela. Facultad de Ciencias de la Educación. España.

Arcaya, H. (2008). Influencias de la comunicación no verbal en los estilos de enseñanza y en los estilos de aprendizaje. [Tesis de Grado]. Universidad de Alicante de España, Facultad de educación, España.

Berlo, D. (1979). El Proceso de Comunicación. Argentina: Ateneo.

Cerezo, M. (2012). Capacidad de comunicación corporal del docente y motivación para el aprendizaje que presentan los alumnos del nivel secundario de la ciudad de Tacna. [Tesis de Grado]. Universidad Nacional Jorge Basadre Grohmann., Facultad de Educación, Tacna.

De la Fuente, J (2002). Perspectivas recientes en el estudio de la motivación, [Tesis de grado]. Universidad de Almería, España.

Delamont, S. (2011). La interacción didáctica. (3 ${ }^{\mathrm{a}}$. Ed.). Madrid: Cincel-Kapelusz

Ekman, P. \& Friesen, W. V. (1969). El repertorio de no verbal Categorías: comportamiento, orígenes, de uso y de codificación. España: Semiótica.

Fiske, J. (1982). Introducción al estudio a la comunicación. ( $1^{a}$ edición). Inglaterra: Norma.

García, E. (2014). Estudio bidireccional de la comunicación no verbal en el aula de Primaria. [Tesis de grado]. Universidad de Valladolid. España.
Gonzales, B. (2003). Factores determinantes el bajo rendimiento académico en educación secundaria. [Tesis de doctorado]. Universidad Complutense de Madrid, Facultad de educación, España.

Günther Rebel. (1995). El lenguaje corporal. Lo que decimos a través de nuestras actitudes, gestos y posturas. Madrid: EDAF.

Gutiérrez, J. (2007). El comportamiento no verbal en el aula. Revista Scielo. Recuperado el 05 $\mathrm{set}$ i e m bre $2016, \quad \mathrm{de}$ : http://www.scielo.org.ve/scielo.php?script=sci _arttext\&pid=S0798-43242007000100010

Hervás, G. (1999). ¿Cómo dominar la comunicación verbal y no verbal? Teoría y actividades. Madrid: Playor.

Keil, G. (2011). Comunicación en el ámbito escolar en relación con los procesos de enseñanza y aprendizaje. [Tesis de grado]. Universidad Abierta Interamericana, Facultad de Investigación y desarrollo Educativo, Argentina.

Kishimoto, B. (2010). La esfera de la comunicación como paradigma de nuevos aprendizajes. [Tesis de Grado]. Universidad Pontificia Católica del Perú, Facultad de ciencias de la comunicación, Lima.

Miller, P.W. (2005): El lenguaje corporal: Una introducción ilustrada por teacher. ( $2^{\mathrm{a}}$ edición). Chicago: Patrick.W. Miller y Asociados.

Pradelli, Á. (2011). La búsqueda del lenguaje: experiencias de transmisión. ( $1^{\circ}$ edición), Buenos Aires: Paidós 